\title{
Review of the radiographic modalities used during dental implant therapy
} - A narrative

SADJ March 2021, Vol. 76 No. 2 p84 - p90

KR Beshtawi', MT Peck², M Chetty ${ }^{3}$

\section{ABSTRACT}

The introduction of digital $\mathrm{x}$-ray receivers which replaced conventional films was a significant radiographic development that is commonly used in daily dental practice. Dental implant therapy (DIT) is a sought after dental therapeutic intervention and dental radiography is an essential component contributing to the success of treatment. Dental radiographs taken in daily practice are generally conventional two-dimensional images and/or three-dimensional images. Ideally, the choice of radiographic technique should be determined after a thorough clinical examination and comprehensive consideration of the advantages, indications, and drawbacks.

Digital three-dimensional modalities that have emerged over the last decade have been incorporated into DIT with the assumption that treatment outcomes will be improved. These modalities are constantly being reassessed and improved but there is a paucity of published information regarding the assessment of variables such as dosages and dimensional accuracy, suggesting that further research in these matters is necessary. This is crucial in order to obtain evidence-based information that may influence future radiographic practices.

In this narrative, the authors present the most commonly used dental radiographic modalities currently used in DIT.

\section{Keywords}

Dental implant, CBCT, panoramic radiograph, periapical radiograph, radiographic assessment, modalities used.

\section{Author affiliations:}

1. Khaled R Beshtawi: B.D.S, MSc, PhD., Department of Oral and Maxillofacial Radiology, Dental Faculty, University of the Western Cape, Cape Town, South Africa.

2. Mogammad T Peck: BChD, MSc, MRD, MChD, FDS RCS, PhD, PGDip Med Ed, MBA, MDT FEd, The City of London Dental School, Southgate College, London, United Kingdom. ORCID Number: 0000-0002-4755-0574

3. Manogari Chetty: $B S c, B C h D, M C h D, P h D$., Department of Craniofacial Biology, Dental Faculty, University of the Western Cape, Cape Town, South Africa.

Corresponding author: Khaled R Beshtawi

Department of Oral and Maxillofacial Radiology, Dental Faculty, University of the Western Cape, Cape Town, South Africa.

Email: kbeshtawi@uwc.ac.za

Author contributions:

1. Khaled R Beshtawi: Conceptualization, analysis, writing: original draft, review and editing - $40 \%$

2. Mogammad T Peck: Conceptualization, writing: review and editing, and supervision - $30 \%$

3. Manogari Chetty: Conceptualization, writing: review and editing, and supervision - $30 \%$

\section{INTRODUCTION}

Dental implant therapy (DIT) is a valuable and highly successful dental intervention that intends to replace missing teeth. ${ }^{1,2}$ This therapy has become increasingly popular and substantial numbers of dental implants are placed and restored every year. ${ }^{3}$

The discovery of X-rays in 1895 by Wilhelm Röntgen, ${ }^{4}$ has revolutionized dental and medical therapies and imaging during various phases of dental implant therapy has a vital role in its success. ${ }^{2,5,6}$ Moreover, the development and transition to digital imaging further enhances the radiographic acumen and offers many advantages such as reduced radiation, immediate acquisition of the radiograph, manipulation of radiographic characteristics like contrast. ${ }^{4}$

Different imaging techniques are used during DIT which have various advantages and disadvantages. The authors present the most common dental radiographic modalities that are currently used during DIT.

\section{CURRENT MAXILLOFACIAL \\ RADIOGRAPHIC TECHNOLOGIES}

Various imaging techniques are employed during dental implant therapy including the conventional two-dimensional examinations namely, intraoral periapical, panoramic, and lateral cephalometric radiographs, and the more sophisticated three-dimensional $\mathrm{x}$-ray volumes such as Computed Tomography (CT), Cone-Beam Computed Tomography (CBCT)). Each radiographic technology has indications, advantages, and disadvantages.

\section{Conventional two-dimensional techniques}

\section{Intraoral periapical radiography (IPR)}

IPR is a widely used imaging technique that exposes a limited number of teeth. ${ }^{7}$ In daily practice, these radiographs are used to evaluate the teeth's architecture, position, boundaries, caries detection, and the status of the periapical regions. ${ }^{7}$ During implant therapy, the IRPs are popular and indicated for potential implant site assessment and during the post-implant assessment. ${ }^{8,9}$

IPR has multiple advantages during implant therapy and is widely available, cost-effective, provides images with high spatial and contrast resolutions, and allows for the assessment of potential implant site boundaries in the vertical and mesiodistal dimensions.5,6,9 Although IPR usually produces only nominal geometrical distortion, 
this geometrical precision can be vary depending on the patients' compliance and operator skills. ${ }^{9}$ Due to the technical challenges, the dimensional accuracy obtained for wide edentulousness bone segments on the IRP can be inconsistent and less reliable. ${ }^{9}$ One of the main disadvantages of the technique is the lack of cross-sectional images of the region of interest. ${ }^{9}$

The lack of this information compromises the optimal assessment of the quality and quantity of the bone structures of the potential implant site, moreover, the anatomical relation with the vital structure in the vicinity of the surgical site may not be accurately revealed. ${ }^{6}$ The estimated effective dose (ED) for a full mouth survey (18-20 radiographs) is $17 \mu \mathrm{Sv}$ (using a CCD sensor). ${ }^{10}$

\section{Lateral cephalometric radiography (LCR)}

LCR is a conventional, two-dimensional radiographic technique that depicts the lateral aspect of the maxillofacial region. ${ }^{9}$ This view provides the clinicians with information about the teeth inclination, jaws relationships, and the soft and hard tissue profile of the patient. ${ }^{11}$

Although the use of LCR during DIT is not very popular, ${ }^{9}$ it may be indicated in order to plan implant treatments in the edentulous midline areas; as this radiograph provides a cross-sectional view of these anterior regions. ${ }^{9,11}$ This view allows a suitable evaluation of the bone quantities in both dimensions (buccolingual and vertical planes of the anterior alveolar ridges), particularly that the LCR has a constant magnification ratio. ${ }^{9}$ Disadvantages of this technique during DIT also exist and include the superimposition of the anatomical structures, teeth and bone, lies in the opposite side of the jaw. Uncertain assessment of bone quality and geometric distortion can be encountered if a patient is incorrectly positioned. ${ }^{5,6,11}$ The estimated ED is 2-6 $\mu \mathrm{Sv}^{10}$

\section{Orthopantomography or panoramic radiography (PAN)}

Panoramic radiographs are a widely used imaging technique that shows a panoramic view of the maxilla and mandible. ${ }^{10}$ This modality is unique as only anatomical structures that lie inside a three-dimensional horseshoeshaped zone, namely the focal trough, are depicted clearly on the radiograph. ${ }^{10}$

Panoramic radiographs are commonly used in various treatment phases during DIT. ${ }^{9}$ These radiographs are indicated during the initial evaluation of the potential implant site and the adjacent structures ${ }^{9,12}$ and frequently prescribed directly after the surgical placement of several implants and during further follow-up. ${ }^{9,13}$

PAN provides a broad view of the jaws, is relatively less expensive, and is widely available. ${ }^{6,9,12,14}$ Nevertheless, these radiographs can be compromised due to geometrical distortion and inherent magnification, reproducibility challenges, uncertainties in bone density assessment, lack of cross-sectional images, inferior resolution compared with intraoral radiographs, and greater technique sensitive. .,6,9,14 $^{-1,4}$

The head position during the acquisition of these radiographs is critical particularly during implant planning as any minor deviation can result in magnification (15-22\%) and image distortion. ${ }^{6,15}$ The ED ranges from 9-24 $\mu \mathrm{Sv} .{ }^{10}$

\section{Three-dimensional radiographic techniques}

\section{Magnetic resonance imaging (MRI)}

MRI is a sophisticated imaging technique that uses a non-ionizing magnetic field and radio waves to reconstruct cross-sectional images. ${ }^{5,16}$ The use of this modality in dental fields including implant therapies are limited, nonetheless, it can be beneficial to use during the planning phase. ${ }^{9}$ During implant therapy, the MRI provides crosssectional images with high soft-tissue contrast and fewer artifacts that allow the radiographic distinction of neurovascular structures, oral mucosa, and the cortical bone.5,9,16

Disadvantages like higher costs, prolonged acquisitions time, challenges of volume interpretation, poor characterization of bone minerals, artifacts from ferromagnetic| metals, and contra-indication for certain patients (e.g. cardiac pacemaker, surgical clips in situ) contributed to its limited use in DIT. 5,9,16

\section{Computed tomography (CT)}

$\mathrm{CT}$ is a three-dimensional imaging technique that was developed by Hounsfield (1972). ${ }^{17}$ This modality improved the diagnostic capability of clinicians in medicine and dentistry. Several developments and multiple generations of the modality have evolved during the last decade which has increased the resultant image qualities. ${ }^{9}$

The CT units generate a fan-shaped X-ray beams that are received by multiple detector arrays where the remaining beam intensities are measured.9,10 These intensity values are incorporated into mathematical algorithms in order to reconstruct multiplanar images. ${ }^{10}$

The use of CT scans during DIT is indicated during the planning phase, in particular, complex cases where the implant site is in close proximity to vital structures and the quantity of the bone is less than optimal. CT scans are also indicated when bone augmentation procedures are needed in sinuses and alveolar ridges, during computerguided surgeries, and in post-operative complications. ${ }^{9,13}$

CT scans are considered advantageous during implant therapy; as this modality provides three-dimensional multi planar views, accurate dimensions, and optimal resolution of the potential implant site and the surrounding structures.9,18 CT scans also allow for reliable quantitative and qualitative bone assessment, vital for the success of DIT, before implant surgery. ${ }^{6,9}$ The main drawbacks of this technique are the generation of high radiation doses compared with conventional radiographs, less availability, higher cost implications, and possible volume artifacts that may arise from metallic objects and patient movement. ${ }^{5,9}$ The estimated ED ranges from 280 to $1410 \mu S_{\text {Sv. }}{ }^{13,19}$

\section{Cone beam computed tomography (CBCT)}

$\mathrm{CBCT}$ is a relatively recent three-dimensional imaging modality that uses a cone-shaped $\mathrm{x}$-ray beam and digital $\mathrm{x}$-ray receivers to reconstruct multiplanar images using special algorithms. ${ }^{9}$ The commercial use of this technique 
in dentistry began in 1999 in Europe and is now a vastly popular imaging modality in the dental practice. ${ }^{9}$

The use of CBCT scans during DIT is indicated during the planning phase especially complex cases involving proximity of vital structures and low bone quantity, in cases where bone augmentation procedures are needed in sinuses and alveolar ridges, during computer-guided surgeries, and in post-operative complications. ${ }^{9,13}$

The use of the modality during DIT has been growing exponentially as it is readily available and easy to use..$^{9,20}$ CBCT's offer fast volumes acquisition (10-80s), lower radiation doses when compared to CT's, high spatial resolution, dimensional accuracy, cheaper unit cost (compared to CT), usually provided with more user-friendly and dentally-oriented software especially when used during implant planning, and the ability to limit the field of view to the region of interest for example just the potential implant site. ${ }^{9,21,22}$ It is worth mentioning that variations in some advantages, volume qualities, and radiation doses of different $x$-ray machines do exist. ${ }^{20}$

CBCT is advantageous in potential implant site assessment as it provides comprehensive anatomical details allowing accurate surgical planning and possible integration with guided surgical techniques. ${ }^{20}$

The drawbacks of CBCT include poor soft-tissue contrast, higher radiation doses when compared with conventional techniques, beam hardening artifact when metallic objects are present, and extra cost implications. ${ }^{9}$ The estimated ED ranges from 19-1,073 $\mu$ Sv and is influenced by the field of view and individual units' dose parameters. ${ }^{10,13}$

\section{PHASES OF DENTAL IMPLANT THERAPY WHERE RADIOGRAPHIC MODALITIES ARE INDICATED}

The quality and quantity of the anatomical details gathered via dental imaging of the potential implant site influences the success of DIT. ${ }^{2}$ Information on the jaw bone anatomy, the quantity and the quality of the alveolar ridge, detection of underlying pathologies, and demarcation of the vital anatomical structure in the vicinity of the implant site can be acquired. 5,6,12,15

Various factors influence the selection of a suitable imaging technique during DIT. These include the amount of anatomical detail required for the treatment, the amount of information gathered through clinical evaluation of the patient, variations in the clinical judgments among clinicians, radiation concerns, and patient-related factors such as esthetic demands and complications risk assessment. ${ }^{11,23}$

The authors further depict the most common radiographic modalities used in the different stages of DIT.

\section{Radiographic examination:}

Planning phase

Thorough planning is a prerequisite for successful dental implant treatment and this decreases the risk of potential postoperative complications. During this stage of treat- ment, the clinician acquires pre-operative vital clinical information on the potential implant site.

Dental imaging plays a major role during this phase as it provides information relating to the potential implant site which includes the alveolar ridge dimensions, the quality of the bone, the spatial relationship of the implant site and other vital structures, determination of the required number of implants, and assessment of the prosthetic needs. ${ }^{5,6}$

Various imaging techniques are being used during this phase including conventional two dimensional to more sophisticated three-dimensional views, ${ }^{2}$ nevertheless, an ideal single imaging modality has not been proposed. ${ }^{9}$ The choice of the type of radiograph to prescribe is often subjective..$^{9}$ Other technical aspects also play a role in this decision and include related costs, accessibility and availability of certain imaging techniques, and radiation exposure levels. ${ }^{6}$

The Intraoral periapical radiographs (IRP) are very commonly used during this phase to initially assess the potential implant sites, appreciation of vital structures, and the discovery of any pathologies in the region of interest. 5,6 The geometrical precision of this type of examination is uncertain and greatly depends on patient compliance and operator skills, ${ }^{9}$ making this mode of examination precarious if it is the only radiographical mode of examination. The use of radiographic markers is then recommended to calibrate and enhance the accuracy of measurements particularly in the vertical plane. ${ }^{9}$

Panoramic radiographs are another example of widespread two-dimensional examinations utilized during this phase. Several published reports indicate the beneficial use of panoramic radiographs during implant treatment $\mathrm{s}^{24-26}$ and condone it even as a single radiographic mode of examination. ${ }^{27}$ The leading prescription of panoramic examinations during implant therapy is documented in multiple surveys conducted in various geographical parts of the world. ${ }^{28-32}$

These examinations are considered simple, widely available, less costly, and expose the patients to only low radiation doses (compared with CT/CBCT). ${ }^{24-26}$ In contrast, single panoramic examinations during this phase are not recommended by other reports ${ }^{33-35}$ due to discrepancies found during the assessment of vertical dimensions on the images. The reliability of the dimensions obtained in panoramic radiographs is influenced by the proper patient positioning during the acquisition. ${ }^{35}$

One of the most important pitfalls of planning implant placement on panoramic radiographs is the inconsistency of the vertical and horizontal magnification factors in various segments of the jaw. ${ }^{35,36}$ Often, within the domain of the focal trough layer, only certain points are found to reveal distortion-free images of the anatomical structure. ${ }^{35}$

Challenges in the interpretation of volume, increased costs, longer acquisition times, and limited availability hinder the wide use of MRI during implant planning. ${ }^{9} \mathrm{Al}-$ though its use is limited, when it is considered for implant planning the use of T1-weighted sequences is recommended. ${ }^{16}$ 
During the planning of implants on the T1 sequence, the cortical bone presents with low signal i.e. appears dark in contrast to the adjacent spongy bone which appears brighter. ${ }^{16} \mathrm{MRI}$ is advantageous during treatment planning in cases where the identification of the neurovascular bundles was not precisely identified using other radiographic techniques. This modality offers higher softtissue contrast and is capable of portraying the neurovascular bundles within their canals. ${ }^{16}$

During the last decade, CBCT has become increasingly employed during several dental procedures in particular during implant planning, especially in that it exposes the patients to lower radiation doses when compared to the traditional CT. ${ }^{20,37-39}$ A survey conducted in the United States (2016) showed that the use of the CBCT was a commonly prescribed method of radiographic investigation during implant planning in academic and private sectors, $49.6 \%$ and $59.1 \%$ respectively. ${ }^{40}$

The use of CBCT during implant planning is advantageous as this imaging technique delivers distortion and superimposition free multi-planar images that allow for precise assessment and measurements of the potential implant sites. ${ }^{11,22}$ Using three-dimensional imaging e.g. CBCT during implant planning can improve the treatment outcomes by enhancing the evaluation of the patient's specific anatomy and reduces the potential of jeopardizing the surrounding structures such as perforation of sinuses and cortical borders and injuring the neurovascular structures during surgery. ${ }^{9,41,42}$

CBCT linear measurements are accurate and reliable during the implant planning stage, confirmed in a recent systemic review. ${ }^{22}$ Although submillimeter discrepancies have been reported in many published reports, the authors ${ }^{22}$ have provided confirmation that the $2 \mathrm{~mm}$ safety margin must be employed, as ranges of over and underestimation of the measurements are also reported. ${ }^{22}$

If computer-guided implant surgery is indicated, threedimensional examinations such as CBCT and/or CT are mandatory. ${ }^{43}$ The surgical and prosthetic phases of implant treatment can be virtually simulated by integrating CBCT and/or CT volumes in the implant planning software. ${ }^{44}$ Interactive virtual implant surgery can be simulated and adjusted the quantity of the available bone, circumvent vital structures, and predict prosthetic and esthetic needs. ${ }^{44,45}$

\section{Overview of bone quality}

The density of bone at the potential implant site is considered one of the vital factors affecting the success of the treatment. ${ }^{46-48}$ Bone quality is not only confined to the density or the mineral content, but includes aspects like the internal architecture of the bone, the alignment of the trabeculae, and matrix-related properties. ${ }^{21,48}$

In CT scans, the tissue densities are represented in Hounsfield units $(\mathrm{HU})$ that are considered a reliable measure to assess the bone density at the candidate implant sites. ${ }^{5,49-51}$ In CBCT volumes, the evidence on the reliability of using CBCT gray values for assessment of bone density is uncertain particularly since these values are not absolute as compared to the CT's HU. ${ }^{21,52,53}$
Discrepancies are reported between the CT's HU values and CBCT's gray values ${ }^{54-57}$ and on the contrary, other reports ${ }^{58,59}$ conclude the opposite. However, the efficacy of using conversion factors to convert the gray values into $\mathrm{HU}$ values is also reported. ${ }^{52,60-62}$

Generally, the CBCT gray values are generated in an arbitrary and predetermined fashion by the manufacturers. ${ }^{52,61}$ An obstacle is the absence of a standard scaling system among the CBCT manufacturers to standardize these gray values, consequently, the interpretation and comparison of these values acquired from different CBCT units will be difficult and impractical in certain instances. ${ }^{21,61}$ The resultant CBCT gray values are greatly influenced by the exposure parameters, machine specifications, and object positions within the $\mathrm{x}$-ray units. ${ }^{57}$

\section{Radiographic examinations:}

Surgical phase (Intra-operative)

Imaging during this phase of treatment i.e. during and directly after surgery, is indicated to confirm the accurate placement of the implant within the planned surgical site and to ensure an ideal position for the prosthetic restoration to follow. 5,6

Conventional two-dimensional images are commonly used during this phase such as Periapical and panoramic radiographs, though periapical radiographs are usually considered adequate for this stage.6,13

The justification to use CBCT in this phase according to the American Academy of Oral and Maxillofacial Radiology (AAOMR) is confined to cases where there is an alteration in the patient's sensory perception and implant mobility. ${ }^{9}$

\section{Radiographic examinations:}

Restorative phase

In this stage, the functional restoration is fabricated and integrated with the implant. Periapical radiographs are commonly utilized in this phase and aid in the assessment of the osteointegration of the fixture with the surrounding bone.

This radiograph also contributes as a baseline radiograph, particularly for future bone attachment level comparisons, and the evaluation of the mechanical integration of the different implant components. ${ }^{63}$ Moreover, optimum elimination of the peri-implant excess of the cementing material can be confirmed using periapical radiographs; the presence of these materials may result in peri-implant complications. ${ }^{63,64}$

It has also been reported that digital periapical radiographs revealed a greater potential in which misfit of the implant-abutment surface could be detected when compared to the analogue counterparts. ${ }^{65}$ In addition, the vertical angle of the $x$-ray beam is found to significantly influence the radiographic evaluation of the implant components where the misfit may be superimposed depending on the angle of the beam. ${ }^{63,66}$

The attainment of a parallel relationship between the x-ray receiver and the implant long axis inside the bone is vital and can be achieved using $x$-ray film holder. ${ }^{63}$ 
Radiographic examinations:

Maintenance phase (Post-prosthetic)

This phase commences directly after the completion of the prosthetic phase and lasts throughout life as long as the implant is present in the patient's mouth. ${ }^{5,6}$

Radiographs are indicated in this phase to ensure and monitor peri-implant osteointegration, evaluate the bone levels, and assess the overall status of the implant, restoration, and surrounding periodontium. ${ }^{5,9}$

Conventional two-dimensional imaging techniques like intraoral periapical radiograph (IPR) or panoramic radiographs especially in instances where the patient received multiple implants, are recommended by AAOMR to suffice this phase of treatment. ${ }^{9}$ IRP is considered more advantageous to evaluate the peri-implant region compared to CBCT; as the metallic nature of the implant body causes radiographic artifacts (i.e. beam hardening) in the resultant CBCT volumes, which hinders the precise evaluation of the region of interest. ${ }^{9}$

Although in most cases conventional imaging is sufficient for the assessment, in the presence of complications related to the procedure like alteration in the sensation and persistent maxillary sinus infections, the European Association for Osseointegration (E.A.O) justifies using CBCT for further investigation. ${ }^{13}$

From a radiographic viewpoint, a marginal bone loss of 0.9-1.6 $\mathrm{mm}$ around the implant during the first year of restoration and less than $0.2 \mathrm{~mm}$ in the following successive years is considered a marker of successful treatment. ${ }^{67-69}$ A reliable evaluation considers several factors that influence the radiographic assessment of marginal bone attachment levels on IPR.

This includes the position of $x$-ray receiver, the $x$-ray beam angulation, the position of the implant in the buccolingual plane, and the possible distortions in the interproximal bony margins on the radiographs. ${ }^{63,67,70}$ For that reason, strict compliance to achieve an ideal parallel relationship between the film and the long axis of the implant is mandatory to obtain reliable radiographs. ${ }^{63,67}$

Additionally, a clinician should consider the limitation of two-dimensional radiographs as they do not reveal the status of bony structures that lie in the buccal and lingual/palatal aspect of the implants. ${ }^{63,67}$

\section{EMERGING TECHNOLOGIES}

\section{Guided implant surgery (GIS)}

Guided implant surgery is a relatively recent method that allows pre-operative virtual simulation of the various phases of dental implant therapy using special software. This simulation is then transferred into the surgical site within the mouth using surgical drilling guides or templates printed in using three-dimensional printers. ${ }^{43,71}$

For this approach to be successful, three-dimensional volumes i.e. CT or CBCT have to be available in order to be integrated into the implant planning software. ${ }^{43,71}$
The inherent artifacts particularly streaking artifact due to densely radiopaque materials may hinder the accurate presentation of the teeth surfaces in these 3D volumes. This issue can be solved by aligning or combining the $\mathrm{x}$-ray volumes with its counterpart intra-oral or a stone model surface scan of the teeth and surrounding oral mucosa. ${ }^{43,72}$

The clinical efficacy of the use of GIS has been reported. ${ }^{73-77}$ For optimal success, the virtual planning requires to be accurately transferred to the patient's mouth which ideally should match the planned dimensions. ${ }^{43}$ For this reason, ensuring proper alignment in-between $3 \mathrm{D}$ volumes and the model scans is indispensable to circumvent inherent imprecision in the resultant surgical template. ${ }^{43}$

\section{DISCUSSION AND CONCLUSION}

Various imaging techniques are being used during different phases of dental implant therapy. The selection of a certain radiographic examination should be done after a thorough clinical evaluation of the patient including dental and medical history. Radiographic modalities used during implant therapy vary in the indications, advantages, and disadvantages offered, considering these factors aids in the appropriate selection of the examination that suffices the phase of the treatment.

The three-dimensional views are advantageous during DIT, but still yields a considerable amount of radiation compared to conventional counterparts, ${ }^{78}$ which is a concern since this modality is fast becoming a routine and popular procedure in various parts of the world. ${ }^{38,79}$ Continuous updates on the most recent radiographic techniques, dimensional accuracy of radiographic modalities, and radiation doses would assist the radiation authorities to establish imaging protocols that ensure clinical efficacy and expose the patient to the least radiation doses.

\section{Declaration of interests}

The authors declare no conflict of interest.

\section{Acknowledgments}

This research is part of a Ph.D. study that is funded by the Research Senate, Faculty of Dentistry, University of the Western Cape, South Africa.

\section{References}

1. Moraschini V, da Poubel LC, Ferreira V, dos Barboza ES. Evaluation of survival and success rates of dental implants reported in longitudinal studies with a follow-up period of at least 10 years: a systematic review. Int J Oral Maxillofac Surg. 2015; 44(3): 377-88.

2. Tyndall DA, Brooks SL. Selection criteria for dental implant site imaging: a position paper of the American Academy of Oral and Maxillofacial radiology. Oral Surg Oral Med Oral Pathol Oral Radiol Endod. 2000 May; 89(5): 630-7.

3. Boyce RA, Klemons G. Treatment Planning for Restorative Implantology. Dent Clin NA. 2015; 59: 291-304.

4. Shah N, Bansal N, Logani A. Recent advances in imaging technologies in dentistry. World J Radiol. 2014 Oct 28; 6(10): $794-807$.

5. Nagarajan A, Perumalsamy R, Thyagarajan R, Namasivayam A. Diagnostic imaging for dental implant therapy. J Clin Imaging Sci. 2014; 4 (Suppl 2): 4.

6. Gupta S, Patil N, Solanki J, Singh R, Laller S. Oral Implant Imaging: A Review. Malays J Med Sci. 2015; 22(3): 7-17. 
7. Gupta A, Devi P, Srivastava R, Jyoti B. Intra oral periapical radiography-basics yet intrigue: A review. Bangladesh $J$ Dent Res Educ. 2014; 4(2): 83-7.

8. Deshpande A, Bhargava D. Intraoral Periapical Radiographs with Grids for Implant Dentistry. J Maxillofac Oral Surg. 2014 Dec; 13(4): $603-5$.

9. Tyndall DA, Price JB, Tetradis S, Ganz SD, Hildebolt C, Scarfe WC, et al. Position statement of the American Academy of Oral and Maxillofacial Radiology on selection criteria for the use of radiology in dental implantology with emphasis on cone beam computed tomography. Oral Surg Oral Med Oral Pathol Oral Radiol. 2012 Jun; 113(6): 817-26.

10. White SC, Pharoah MJ. Oral radiology: principles and interpretation. $7^{\text {th }}$ edition. St. Louis, Missouri: Mosby, Elsevier; 2013; 86-7.

11. Agrawal A, Agrawal G, Nagarajappa A, Sreedevi K, Kakkad A. Journey from 2D to 3D: Implant imaging a review. Int Contemp Dent Med Rev. 2014.

12. Lingam A, Reddy L, Nimma V, Pradeep K. "Dental implant radiology" - Emerging concepts in planning implants. J Orofac Sci. 2013; 5(2): 88.

13. Harris D, Horner K, Gröndahl K, Jacobs R, Helmrot E, Benic GI, et al. E.A.O. guidelines for the use of diagnostic imaging in implant dentistry 2011. A consensus workshop organized by the European Association for Osseointegration at the Medical University of Warsaw. Clin Oral Implants Res. 2012 Nov; 23(11): 1243-53.

14. Manisundar N, Saravanakumar Hemalatha BV, Manigandan T, Amudhan A. Implant Imaging-A Literature Review. Biosci Biotechnol Res ASIA. 2014; 11(1): 179 - 87.

15. Karjodkar FR. Textbook of Dental and Maxillofacial Radiology. $2^{\text {nd }}$ ed. New Delhi (IND): Jaypee Brothers; 2009. 881-928.

16. Gray CF, Redpath TW, Smith FW, Staff RT. Advanced imaging: Magnetic resonance imaging in implant dentistry. Clin Oral Implants Res. 2003 Feb;14(1):18-27.

17. Hounsfield G. Computerized transverse axial scanning (tomography): Part I description of system. Br J Radiol. 1973 ;46(552): 1016-22.

18. Seeram E. Computed tomography: physical principles, clinical applications, and quality control. $3^{\text {rd }}$ ed. St. Louis: Saunders Elsevier; 2009.

19. European Commission. Protection Radiation No 172 Cone beam CT for dental and maxillofacial radiology (Evidencebased guidelines). 2012

20. Jacobs R, Salmon B, Codari M, Hassan B, Bornstein MM Cone beam computed tomography in implant dentistry: recommendations for clinical use. BMC Oral Health. 2018 18(1): 88.

21. Sahai S. Recent advances in imaging technologies in implant dentistry. J Int Clin Dent Res Organ. 2015; 7(3): 19.

22. Fokas G, Vaughn VM, Scarfe WC, Bornstein MM. Accuracy of linear measurements on CBCT images related to presurgical implant treatment planning: A systematic review. Clin Oral Implants Res. 2018; 29(Suppl 16): 393-415.

23. Bornstein M, Scarfe W, Vaughn V, Jacobs R. Cone Beam Computed Tomography in Implant Dentistry: A Systematic Review Focusing on Guidelines, Indications, and Radiation Dose Risks. Int J Oral Maxillofac Implants. 2014 Jan; 29 (Supplement): 55-77.

24. Vazquez L, Saulacic N, Belser U, Bernard JP. Efficacy of panoramic radiographs in the preoperative planning of posterior mandibular implants: A prospective clinical study of 1527 consecutively treated patients. Clin Oral Implants Res. 2008; 19(1): 81-5.

25. Kim YK, Park JY, Kim SG, Kim JS, Kim JD. Magnification rate of digital panoramic radiographs and its effectiveness for pre-operative assessment of dental implants. Dentomaxillofacial Radiol. 2011; 40(2): 76-83.

26. Assaf M, Gharbyah A. Accuracy of Computerized Vertical Measurements on Digital Orthopantomographs: Posterior Mandibular Region. J Clin Imaging Sci. 2014; 4(2): 7.
27. Devlin H, Yuan J. Object position and image magnification in dental panoramic radiography: A theoretical analysis. Dentomaxillofacial Radiol. 2013 Jan; 42(1): 29951683.

28. Sakakura C, Morais J, Loffredo L, Scaf G. A survey of radiographic prescription in dental implant assessment. Dentomaxillofacial Radiol. 2003 Nov; 32(6): 397-400.

29. Ramakrishnan P, Shafi FM, Subhash A, Kumara AEG, Chakkarayan J, Vengalath J. A survey on radiographic prescription practices in dental implant assessment among dentists in Kerala, India. Oral Health Dent Manag. 2014 Sep; 13(3): 826-30.

30. Alnahwi M, Alqarni A, Alqahtani R, Baher Baker M, Alshahrani FN. A survey on radiographic prescription practices in dental implant assessment. J Appl Dent Med Sci. 2017; 3(1).

31. Majid I, Mukith ur Rahaman S, Sowbhagya M, Alikutty F, Kumar H. Radiographic prescription trends in dental implant site. J Dent Implant. 2014; 4(2): 140.

32. Rabi H, Qirresh E, Rabi T. Radiographic Prescription Trends among Palestinian Dentists for Dental Implant Placement - A Cross Sectional Survey. J Dent Probl Solut. 2017; 11 - 4.

33. Pertl L, Gashi-Cenkoglu B, Reichmann J, Jakse N, Pertl C. Preoperative assessment of the mandibular canal in implant surgery: comparison of rotational panoramic radiography (OPG), computed tomography (CT) and cone beam computed tomography (CBCT) for preoperative assessment in implant surgery. Eur J Oral Implantol. 2013; 6(1): 73-80.

34. Lindh C, Petersson A, Klinge B. Measurements of distances related to the mandibular canal in radiographs. Clin Oral Implants Res. 1995; 6(2): 96-103.

35. Riecke B, Friedrich RE, Schulze D, Loos C, Blessmann M, Heiland $\mathrm{M}$, et al. Impact of malpositioning on panoramic radiography in implant dentistry. Clin Oral Investig. 2015; 19(4): 781-90.

36. Gomez-Roman G, Lukas D, Beniashvili R, Schulte W. Areadependent enlargement ratios of panoramic tomography on orthograde patient positioning and its significance for implant dentistry. Int J Oral Maxillofac Implants. 1999; 14(2): 248-57.

37. Deeb G, Antonos L, Tack S, Carrico C, Laskin D, Deeb JG. Is Cone-Beam Computed Tomography Always Necessary for Dental Implant Placement? J Oral Maxillofac Surg. 2017 Feb; 75(2): 285-9.

38. Noffke C, Farman A, Nel S, Nzima N. Guidelines for the safe use of dental and maxillofacial CBCT: a review with recommendations for South Africa. SADJ. 2011; 66(6): 264-6.

39. Chau ACM, Fung K. Comparison of radiation dose for implant imaging using conventional spiral tomography, computed tomography, and cone-beam computed tomography. Oral Surgery, Oral Med Oral Pathol Oral Radiol Endodontology. 2009; 107(4): 559-65.

40. Carter JB, Stone JD, Clark RS, Mercer JE. Applications of Cone-Beam Computed Tomography in Oral and Maxillofacial Surgery: An Overview of Published Indications and Clinical Usage in United States Academic Centers and Oral and Maxillofacial Surgery Practices. J Oral Maxillofac Surg. 2016 Apr; 74(4): 668-79.

41. Kraut RA. Interactive CT diagnostics, planning and preparation for dental implants. Implant Dent. 1998; 7(1): 19-25.

42. Hatcher DC, Dial C, Mayorga C. Cone beam CT for presurgical assessment of implant sites. J Calif Dent Assoc. 2003 Nov; 31(11): $825-33$.

43. Flügge T, Derksen W, te Poel J, Hassan B, Nelson K, Wismeijer D. Registration of cone beam computed tomography data and intraoral surface scans - A prerequisite for guided implant surgery with CAD/CAM drilling guides. Clin Oral Implants Res. 2017 Sep; 28(9): 1113-8.

44. Colombo M, Mangano C, Mijiritsky E, Krebs M, Hauschild U, Fortin T. Clinical applications and effectiveness of guided implant surgery: a critical review based on randomized controlled trials. BMC Oral Health. 2017 Dec 13; 17(1): 150. 
45. Grunder U, Gracis S, Capelli M. Influence of the 3-D boneto-implant relationship on esthetics. Int J Periodontics Restorative Dent. 2005 Apr; 25(2): 113-9.

46. Wood MR, Vermilyea SG, Committee on Research in Fixed Prosthodontics of the Academy of Fixed Prosthodontics. A review of selected dental literature on evidence-based treatment planning for dental implants: report of the Committee on Research in Fixed Prosthodontics of the Academy of Fixed Prosthodontics. J Prosthet Dent. 2004 Nov; 92(5): 447-62.

47. Drage NA, Palmer RM, Blake G, Wilson R, Crane F, Fogelman I. A comparison of bone mineral density in the spine, hip and jaws of edentulous subjects. Clin Oral Implants Res. 2007 Aug; 18(4): 496-500.

48. Lindh C, Obrant K, Petersson A. Maxillary bone mineral density and its relationship to the bone mineral density of the lumbar spine and hip. Oral Surg Oral Med Oral Pathol Oral Radiol Endod. 2004 Jul; 98(1): 102-9.

49. Lindh C, Nilsson M, Klinge B, Petersson A. Quantitative computed tomography of trabecular bone in the mandible. Dentomaxillofacial Radiol. 1996 Jun; 25(3): 146-50.

50. Turkyilmaz I, Tözüm TF, Tumer C. Bone density assessments of oral implant sites using computerized tomography. J Oral Rehabil. 2007; 34(4): 267-72.

51. Aksoy U, Eratalay K, Tözüm TF. The possible association among bone density values, resonance frequency measurements,tactile sense, and histomorphometric evaluations of dental implant osteotomy sites: A preliminary study. Implant Dent. 2009; 18(4): 316-25.

52. Cassetta M, Stefanelli LV, Pacifici A, Pacifici L, Barbato E. How Accurate Is CBCT in Measuring Bone Density? A Comparative CBCT-CT In Vitro Study. Clin Implant Dent Relat Res. 2014 Aug; 16(4): 471-8.

53. Arisan V, Karabuda ZC, Avsever H, Özdemir T. Conventional Multi-Slice Computed Tomography (CT) and Cone-Beam CT (CBCT) for Computer-Assisted Implant Placement. Part I: Relationship of Radiographic Gray Density and Implant Stability. Clin Implant Dent Relat Res. 2013 Dec; 15(6): 893-906.

54. Katsumata A, Hirukawa A, Okumura S, Naitoh M, Fujishita M, Ariji $E$, et al. Effects of image artifacts on gray-value density in limited-volume cone-beam computerized tomography. Oral Surgery, Oral Med Oral Pathol Oral Radiol Endodontology. 2007 Dec; 104(6): 829-36.

55. Armstrong RT. Acceptability of Cone Beam CT vs. Multi-Detector CT for 3D Anatomic Model Construction. J Oral Maxillofac Surg. 2006 Sep 1; 64(9): 37.

56. Miles D, Danforth R. A Clinician's Guide to Understanding Cone Beam Volumetric Imaging (CBVI). Peer-Reviewed Publication - Academy of Dental Therapeutics and Stomatology. 2008.

57. Nackaerts O, Maes F, Yan H, Couto Souza P, Pauwels R, Jacobs R. Analysis of intensity variability in multislice and cone beam computed tomography. Clin Oral Implants Res. 2011 Aug; 22(8): $873-9$

58. Naitoh M, Hirukawa A, Katsumata A, Ariji E. Evaluation of voxel values in mandibular cancellous bone: relationship between cone-beam computed tomography and multislice helical computed tomography. Clin Oral Implants Res. 2009 May; 20(5): 503-6.

59. Nomura Y, Watanabe H, Honda E, Kurabayashi T. Reliability of voxel values from cone-beam computed tomography for dental use in evaluating bone mineral density. Clin Oral Implants Res. 2010 May; 21(5): 558-62.

60. Reeves T, Mah P, McDavid W. Deriving Hounsfield units using grey levels in cone beam CT: a clinical application. Dentomaxillofacial Radiol. 2012 Sep; 41(6): 500-8.

61. Mah P, Reeves TE, McDavid WD. Deriving Hounsfield units using grey levels in cone beam computed tomography. Dentomaxillofacial Radiol. 2010 Sep; 39(6): 323-35.
62. Lagravère M, Fang Y, Carey J, Toogood R, Packota G, Major P. Density conversion factor determined using a conebeam computed tomography unit NewTom QR-DVT 9000. Dentomaxillofacial Radiol. 2006 Nov; 35(6): 407-9.

63. Wadhwani CPK, Schuler R, Taylor S, Chen CSK. Intraoral radiography and dental implant restoration. Dent Today. 2012 Aug; 31(8): 66, 68, 70-1; quiz 72-3.

64. Pauletto N, Lahiffe BJ, Walton JN. Complications associated with excess cement around crowns on osseointegrated implants: a clinical report. Int J Oral Maxillofac Implants. 1999; 14(6): $865-8$.

65. Oliveira B, Valerio C, Jansen W, Zenóbio E, Manzi F. Accuracy of Digital Versus Conventional Periapical Radiographs to Detect Misfit at the Implant-Abutment Interface. Int J Oral Maxillofac Implants. 2016 Sep; 31(5): 1023-9.

66. Begoña Ormaechea M, Millstein P, Hirayama H. Tube angulation effect on radiographic analysis of the implant-abutment interface. Int J Oral Maxillofac Implants. 1999; 14(1): 77-85.

67. Cassetta M, Di Giorgio R, Barbato E. Are intraoral radiographs reliable in determining peri-implant marginal bone level changes? The correlation between open surgical measurements and peri-apical radiographs. Int J Oral Maxillofac Surg. 2018; 47(10): 1358-64.

68. Albrektsson T, Zarb G, Worthington P, Eriksson AR. The longterm efficacy of currently used dental implants: a review and proposed criteria of success. Int J Oral Maxillofac Implants. 1986; 1(1): 11-25.

69. Smith DE, Zarb GA. Criteria for success of osseointegrated endosseous implants. J Prosthet Dent. 1989; 62(5): 567-72.

70. Sewerin I. Errors in radiographic assessment of marginal bone height around osseointegrated implants. Eur J Oral Sci. 1990; 98(5): 428-33.

71. Flügge TV, Nelson K, Schmelzeisen R, Metzger MC. ThreeDimensional Plotting and Printing of an Implant Drilling Guide: Simplifying Guided Implant Surgery. J Oral Maxillofac Surg. 2013 Aug; 71(8): 1340-6.

72. Plooij JM, Maal TJJ, Haers P, Borstlap WA Kuijpers-Jagtman AM, Bergé SJ. Digital three-dimensional image fusion processes for planning and evaluating orthodontics and orthognathic surgery. A systematic review. Int $J$ Oral Maxillofac Surg. 2011 Apr; 40(4): 341-52.

73. Shen P, Zhao J, Fan L, Qiu H, Xu W, Wang Y, et al. Accuracy evaluation of computer-designed surgical guide template in oral implantology. J Cranio-Maxillofacial Surg. 2015 Dec; 43(10): 2189-94.

74. Filius MAP, Kraeima J, Vissink A, Janssen KI, Raghoebar GM, Visser A. Three-dimensional computer-guided implant placement in oligodontia. Int J Implant Dent. 2017 Dec 8; 3(1): 30.

75. Platzer S, Bertha G, Heschl A, Wegscheider WA, Lorenzoni M. Three-Dimensional Accuracy of Guided Implant Placement: Indirect Assessment of Clinical Outcomes. Clin Implant Dent Relat Res. 2012 Jan; 15(5).

76. Vercruyssen M, Cox C, Coucke W, Naert I, Jacobs R, Quirynen M. A randomized clinical trial comparing guided implant surgery (bone- or mucosa-supported) with mental navigation or the use of a pilot-drill template. J Clin Periodontol. 2014 Jul; 41(7): 717-23.

77. Behneke A, Burwinkel M, Behneke N. Factors influencing transfer accuracy of cone beam CT-derived template-based implant placement. Clin Oral Implants Res. 2012 Apr; 23(4): $416-3$.

78. Davies J, Johnson B, Drage N. Effective doses from cone beam CT investigation of the jaws. Dentomaxillofacial Radiol. 2012 Jan; 41(1): 30-6.

79. Li G. Patient radiation dose and protection from cone-beam computed tomography. Imaging Sci Dent. 2013 Jun; 43(2): 63. 